\title{
WhatsApp como herramienta de verificación de fake news. El caso de B de Bulo
}

\author{
WhatsApp as a verification tool for fake \\ news. The case of B de Bulo
}

\begin{abstract}
Bella Palomo [CV] [ Orcid] [ $\mathrm{C} G \underline{G}$ ] Catedrática del Departamento de Periodismo. Universidad de Málaga (UMA), España. bellapalomo@uma.es

Jon Sedano [CV] [- Orcid]. Doctorando. Facultad de Ciencias de la Comunicación. Universidad de Málaga (UMA), España. jon@uma.es
\end{abstract}

\begin{abstract}
s
[ES] Introducción. España es el país europeo con mayor penetración de WhatsApp para el consumo de noticias, según el Digital News Report 2017. Hipótesis y objetivos. Adoptar esta herramienta de comunicación inmediata permite a los medios incrementar cualitativamente las fuentes disponibles, el tráfico e incluso involucrar a las audiencias activas en tareas de verificación. Los objetivos planteados consisten en describir el proceso de creación de la sección B de Bulo en el periódico Sur, analizar su funcionamiento y repercusión en redes sociales. Metodología. Se aplica una metodología mixta, que combina técnicas cuantitativas y cualitativas: análisis de contenido, entrevistas con responsables de la gestión del servicio, observación participante y monitorización de visitas. Resultados. La publicación de contenidos sobre bulos se realiza cada 48 ó 72 horas, y se evitan aquellos relacionados con niños o política. A pesar de estas limitaciones editoriales, los textos de esta sección forman parte de la lista de los contenidos más leídos. Conclusiones. Los resultados confirman el éxito de la relación de proximidad que se establece entre la redacción y los lectores vía WhatsApp.

[EN] Introduction. According to the Digital News Report 2017, Spain is the European country with the highest penetration of WhatsApp for news consumption. Hypothesis and objectives. This tool of immediate communication allows media to increase from a qualitative perspective sources, traffic and even involve active audiences in fact-checking tasks. Our goals are to describe the process of creating B of Bulo feature in the newspaper Sur, analyze its operation and impact on social networks. Methodology. A mixed methodology is applied, by combining quantitative and qualitative techniques: content analysis, interviews with those responsible for the management of the service, participant observation and monitoring of visits. Results. The publication of content on hoaxes is done every 48 or 72 hours, and those related to children or politics are avoided. Despite these editorial limitations, news pieces in this section are part of the list of the most read contents. Conclusions. The results confirm the success of the proximity relationship established between newsroom and audiences via Whats App.
\end{abstract}




\section{Keywords}

[ES] noticias falsas; posverdad; verificación; prensa local; Telegram; WhatsApp.

[EN] Fake news; post-truth; fact-checking; local press; Telegram; WhatsApp.

\section{Contents}

[ES] 1. Introducción. 2. Objetivos y metodología. 2.1. Estrategias metodológicas. 3. Resultados. 3.1. Rutinas productivas. 3.2. Estrategias de verificación. 3.3. La clave del éxito. 4. Discusión y conclusiones. 5. Referencia bibliográficas.

[EN]: 1. Introduction. 2. Objectives and methodology. 2.1. Methodological strategies. 3. Results. 3.1. Productive routines. 3.2. Fact-checking strategies. 3.3. Sucessfull key. 4. Conclusion and discussion. 5. References.

Translation of paper by Yuhanny Henares (Academic translator, Universitat de Barcelona)

\section{Introducción}

Las noticias falsas of fake news se han convertido en la actualidad en uno de los principales problemas de la Era Digital. Su relevancia obligó a que el diccionario de la Real Academia incorporara en 2017 el término postverdad, que alude a la "distorsión deliberada de una realidad, que manipula creencias y emociones con el fin de influir en la opinión pública y en actitudes sociales". La circulación intensiva de este tipo de contenidos requiere la reacción de los periodistas para proteger y defender la calidad y la veracidad de la información profesional.

Que una noticia falsa llegue a la portada de un medio relevante refleja en la mayoría de ocasiones una falta de verificación por los periodistas implicados. El 24 de enero de 2013 El País publicaba en primera página una fotografía exclusiva del presidente Hugo Chávez entubado mientras recibía tratamiento médico en Cuba. Otros medios también recibieron la propuesta de la agencia Gtres Online, la única fuente que tenía ese material, pero solo El País decidió difundirla. La imagen resultó ser falsa. Las exclusivas ofrecen distinción, pero no contrastadas pueden dañar seriamente la credibilidad del medio, y esos fallos favorecen la huida de la audiencia. En este sentido, las redacciones de los cibermedios deben afrontar una doble presión: ser los primeros en difundir una noticia y publicarla con toda la corrección y cuidado de una edición escrita (Torres, 2007). Y son muchos los medios que caen en la tentación de primar la velocidad y el impacto sobre el contraste, sin comprender que el tiempo se convierte en un problema ético cuando se prefiere lo rápido a lo correcto (CdPeriodismo, 2013). Algunos expertos, como la periodista Delia Rodríguez, han advertido de los peligros de estas prácticas: "todo tiene un autor, un culpable que ha encargado el tema, unas manos que lo han ejecutado, aunque no lo firmen. Si copias, si mientes, si manipulas, si escribes basura siempre hay alguien que se va a dar cuenta, porque estamos en Internet y todos somos expertos en algo" (Rodríguez, 2016).

A pesar de estos consejos, que comulgan con los principios básicos de la profesión, El Mundo publicaba un reportaje el 26 de noviembre de 2016 sobre un padre coraje y su enferma hija Nadia, cuya cura no se podían permitir. El diario ayudó a recaudar casi 150.000 euros, aunque solo aportaron la versión del progenitor. Todo resultó ser una farsa. Fueron otros periodistas (Josu Mezo en el blog Mala Prensa, Ángela Bernardo en Hipertextual, y Manuel Ansede y Elena Sevillano en El País) los que desmontaron la historia e hicieron sus deberes: contrastar fuentes. Esta es por tanto una de las principales debilidades del periodismo español, la escassez o incluso la ausencia de fuentes (Chicote, 2010). 
El periodista Craig Silverman confirma en Verification Handbook (2014) que algo tan básico como comprobar los datos, se está perdiendo en las redacciones, y la facilidad técnica de poder rectificar cualquier información errónea, no debería reducir el proceso de publicación. En este sentido, la Era de la Información ha cambiado el paradigma periodístico. Los medios han perdido el centro y no son los únicos emisores de información (Paniagua, 2017). Las redes sociales, que se han multiplicado en los últimos diez años, ocupan ahora ese espacio, ya han favorecido una hiperactividad comunicativa basada en un exceso de contenidos que genera 'ruido', permitiendo que se cuelen rumores y surjan noticias falsas que tanto periodistas como consumidores no son siempre capaces de distinguir.

Las estrategias implantadas en los medios derivadas de este contexto de inseguridad informativa han sido variadas. La agencia France Press intentó frenar esta situación en 2009 al prohibir a sus redactores el uso de Facebook o Wikipedia como fuente de información. Otros, como Steve Buffy, editor de Gazzete Communications, recomendaba en cambio utilizar la red social de Mark Zuckerberg como punto de partida para la investigación, teniendo eso sí, gran cautela.

Aun así, Facebook está trabajando en mejorar su sistema tras lo ocurrido en 2016, cuando unos ex trabajadores de la red social aseguraron haber 'camuflado' noticias que enaltecían las políticas más conservadoras entre los Trending Topics de Facebook, o lo que es lo mismo, el sistema que la plataforma tenía para mostrar a los usuarios las noticias más destacadas. Zuckerberg realizó entonces lo que meses después se convertiría en uno de los mayores problemas de la red social: la automatización de noticias. En ese momento, el algoritmo no distinguía entre real o falso. De esta forma, la red social comenzó a llenarse de noticias falsas que los usuarios tomaron como ciertas. Todo ello potenciado por el periodo de elecciones estadounidense. Hasta el propio Barack Obama se mostró preocupado por el auge de este tipo de noticias y la imposibilidad que tenían los usuarios para discriminarlas. El II Informe de la Asociación de Internautas Española confirma que al $70 \%$ de los usuarios les cuesta distinguir un bulo de una noticia fiable (Asociación de Internautas, 2009).

Debido a estos antecedentes, en el último lustro se han puesto en marcha varias plataformas dedicadas a detectar este tipo de informaciones falsas. En España por ejemplo nacieron tres en un año: Maldito Bulo en noviembre de 2016, El Tragabulos en enero de 2017 y B de Bulo en marzo de 2017.

Aunque los periodistas están más conectados que nunca, la falta de recursos, tomar decisiones basadas en frases, abreviaturas, emoticonos, o una comunicación fugaz que pueden o no ser representaciones precisas de la verdad, hacen que sea difícil mantener los estándares de calidad que deben caracterizar al periodismo. Una situación que ha provocado que solo un tercio de la población en España confíe en los medios (Reuters Institute Digital News Report, 2015).

Este panorama desolador convive con otra realidad, y es la expansión de la comunicación móvil. La Asociación para la Investigación de los Medios de Comunicación, AIMC, indicaba en 2017 que el 94,3 por ciento de los usuarios de Internet navegan por la web a través de teléfonos inteligentes y que el 62,7 por ciento de los usuarios de Internet lo hace mientras están en la calle. WhatsApp ha convertido a los españoles en los mayores usuarios de su aplicación en Europa, estando presente en el 98 por ciento de los smartphones que hay en el país (Informe CNMC).

En el plano laboral, de acuerdo con una encuesta del CIS, el 25\% de los españoles usa WhatsApp para cuestiones relacionadas con el trabajo. En este contexto, el uso de la comunicación electrónica ha superado la comunicación cara a cara y de voz a voz por un amplio margen. Los estudios demuestran 
que la Generación Y y los Millennials prefieren usar la mensajería instantánea u otras redes sociales en lugar de acudir a una oficina y comunicarse presencialmente.

Durante años, Twitter ha fijado la agenda informativa, pero ahora los medios se enfrentan a una era post-Twitter, porque plataformas como WhatsApp o Telegram han permitido transformar mensajes inmediatos también en noticias, de forma que la audiencia pueda condicionar la agenda (Batra, 2016). Implicar y comprometer a la audiencia activamente en un periodismo colaborativo basado en la comunicación inmediata, experimentando e introduciéndolo en procesos de verificación y filtrado de contenidos, constituye un reto, pero también una oportunidad para incrementar la audiencia y su fidelidad.

Desde 2015, medios como La Sexta Noticias, El Periódico de Cataluña o Sur han incorporado a sus rutinas estos nuevos hábitos de los consumidores, aplicando estrategias respaldadas por datos como que el $69 \%$ de los usuarios que comentan contenidos de televisión envían sus mensajes a través de WhatsApp (Suñé, 2016). En el plano internacional, iniciativas de Reddit, Propublica, The Guardian, BBC o The Washington Post también han demostrado que los medios se pueden beneficiar de las audiencias activas obteniendo más fuentes y comentarios con enlaces a evidencias.

Pese a esta adaptación profesional al entorno de la comunicación inmediada, la circulación de noticias falsas resulta alarmante. El problema no son las nuevas redes, sino no comprobar quién firma el contenido, por lo que hay que buscar, comparar y acudir siempre a marcas fiables, como se hace con la alimentación (Casal, 2016), porque si no, se puede herir de muerte al periodismo. El actor Denzel Washington grabó un vídeo para desmentir el rumor de su apoyo a Trump diciendo: "Si no se lee el periódico se está desinformado, pero si se lee, se está mal informado” (Mèdia.cat, 2016).

En esta misma línea, las noticias falsas publicadas en Facebook llegaron a generar un gran debate, sobre si Trump ganó la presidencia gracias a ellas ya que, según un estudio del Pew Research Center, un $60 \%$ de los estadounidenses obtuvieron información sobre las elecciones mediante esta red social (Gottfried y Shearer, 2016). La respuesta de Zuckerberg consistió en crear un sistema de avisos, similar al Fact-Check de Google, para indicar a los usuarios si una noticia es falsa o no. En el caso del buscador, la opción la deben utilizar los propios medios cuando están plenamente seguros de que han realizado su trabajo de manera responsable. Por otro lado, Facebook está estudiando la posibilidad de seguir confiando en la inteligencia artificial al utilizar un algoritmo que identifica las noticias falsas. Aunque esto puede suponer un nuevo problema a largo plazo: rebasar la estrecha línea que hay entre filtrar y censurar. Paralelamante, un grupo de cuatro jóvenes ha resuelto por ahora el problema de las noticias falsas implementando una extensión para el navegador Chrome (Itkowitz, 2016).

Medios de comunicación en colaboración con centros de investigación también han adoptado un rol activo para protegerse de la circulación de noticias falsas. Iniciativas como The Trust Project, respaldada internacionalmente por medios como La Repubblica, The Economist, La Stampa, The Washington Post o Trinity Mirror fomentan la creación de protocolos y herramientas para identificar y certificar el contenido que hay en la Red. Duke Reporter's Lab ha elaborado también un directorio donde ha localizado 126 sitios activos que se dedican a la verificación de contenidos. Politifact es uno de los más conocidos porque obtuvo un premio Pulitzer.

En España la situación resulta alarmante. El Reuters Institute Digital News Report 2015 indicó que los medios españoles eran los menos creíbles de Europa y los segundos en el mundo, por detrás de los norteamericanos. Estos datos exigen una reacción y una respuesta activa por parte de medios e investigadores, que permita reconducir una actividad comunicativa desacreditada. Desarrollar de 
forma responsable nuevas herramientas o estrategias que faciliten verificar contenidos y detectar rumores resulta fundamental para salvaguardar la credibilidad del medio. En España se han puesto en marcha iniciativas de fact-checking como El Objetivo, Polétika, La Chistera, Maldito Bulo o El Tragabulos Este artículo se centra en analizar una de estas experiencias, B de Bulo.

B de Bulo representa un modelo especial porque esta sección tiene un teléfono móvil para recibir mensajes de la audiencia a través de WhatsApp sobre aquellos contenidos que desean saber si son verdaderos o falsos. Muchos mensajes falsos se transforman en contenido viral que circula a través de WhatsApp, y esta sección selecciona los contenidos falsos que la audiencia podría recibir, los analiza y recurre a diferentes fuentes para explicar por qué el mensaje es un engaño, ofreciendo un servicio útil al ciudadano.

A pesar de la relevancia del fenómeno, desde la academia la literatura al respecto es escasa y reciente. No hay ninguna tesis centrada en el uso que los medios de comunicación hacen de las aplicaciones de mensajería instantánea para la verificación. Hasta el momento, los estudios han abordado principalmente el análisis de ciertas coberturas puntuales (Ramalho, 2014), casos de referencia internacional como The Washington Post o The Guardian (López, Rodríguez y Álvarez, 2016), propuestas metodológicas (Sedano y Palomo, 2018) o el uso que los medios españoles hacen de WhatsApp (Fares, 2018; Negreira, López y Lozano, 2017).

\section{Objetivos y metodología}

Muchos artículos de investigación han explorado la participación ciudadana en los cibermedios. Es más difícil encontrar estudios centrados en la visión interna profesional, describiendo y/o analizando

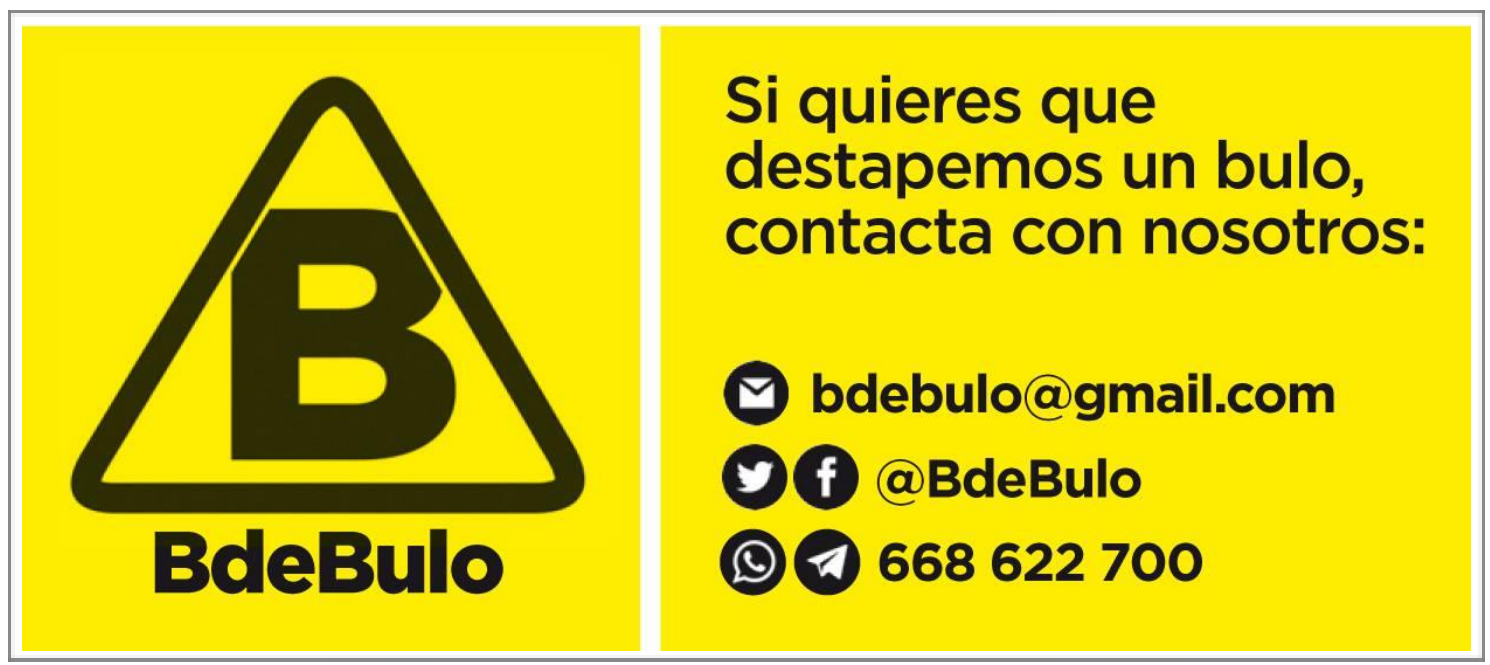

Imagen que acompaña a B de Bulo en redes sociales. SUR

cómo las redacciones manejan la comunicación directa, qué estrategias están desarrollando y cómo gestionan los periodistas estas nuevas rutinas. Estas limitaciones son parte de nuestros objetivos.

Esta investigación posee un triple objetivo: describir el proceso de creación de $B$ de Bulo, sección perteneciente al diario malagueño Sur (Grupo Vocento) fundada en marzo de 2017 en su edición digital; en segundo lugar, analizar su repercusión en redes sociales: cuántos seguidores han alcanzado (Twitter y Facebook), qué noticias han sido más compartidas y cuáles han tenido mayor impacto entre los usuarios. Y, en tercer lugar, se estudiarán las rutinas productivas que rodean a dicha sección, para 
conocer cómo eligen los temas, cómo verifican la información publicada y qué tipo de feedback se establece con los usuarios.

Para abordar dichos objetivos, se aplica una metodología mixta, que combina técnicas cuantitativas y cualitativas: análisis de contenido, entrevistas con responsables del mantenimiento de dicha sección, observación participante y estudios de datos estadísticos.

\subsection{Estrategias metodológicas}

La entrevista en profundidad ofrece evidencias de la naturaleza del fenómeno a estudiar, facilitando el contexto y el origen en el que surge, y se enriquece de la visión interna de quien posee una experiencia directa con la situación a analizar (Miller y Barry, 2016: 51). Por este motivo, en una primera fase se entrevistó a Elena de Miguel, redactora jefe del departamento digital de Diario Sur, lo que permitió conocer qué motivó la creación de la sección $B$ de Bulo, cómo se gestionó, cuánto tiempo llevó preparar su inicio, cuáles son los principales problemas a los que se enfrentan regularmente, qué fuentes utilizan, qué técnicas de verificación emplean y cómo seleccionan los contenidos.

Otro método etnográfico implementado para la recolección de datos fue la observación participante, entendida como la mirada activa que faculta al investigador a aprender acerca de una actividad en el escenario natural donde se desarrolla al involucrarse en la rutina (Kawulich, 2006). En este caso, el investigador era simultáneamente el profesional encargado de implementar la sección, lo que favoreció que los miembros del entorno a analizar actuaran con naturalidad y se evitó la mirada de un intruso que puede derivar en una situación incómoda en el seno de la redacción.

Este abordaje cualitativo se complementó con el análisis de contenido de todas las noticias publicadas durante cuatro meses en la sección $B$ de Bulo desde su fundación el 10 de marzo de 2017 al 10 de julio del mismo año, revisando las temáticas, el uso de las fuentes realizado y categorizando cómo se verifican las noticias o se desmienten los bulos.

En lo que respecta a las redes sociales se ha estudiado su crecimiento tanto en Twitter como en Facebook, las dos plataformas que utiliza. También se ha analizado el alcance en Facebook y sus interacciones para determinar la noticia que ha llegado a más personas, así como la más compartida o con mayor número de reacciones. En Twitter se ha realizado un análisis similar, estudiando los favoritos y retuits obtenidos. Para conseguir estos datos, SUR facilitó el acceso del investigador al sistema de estadísticas que como usuario posee en ambas plataformas.

\section{Resultados}

B de Bulo nace a iniciativa de un becario, con el objetivo de implementar una sección de verificación abierta a la comunidad; una forma de ayudar a los ciudadanos a detectar rumores.

Tras la realización de las entrevistas y tras analizar las 50 noticias (una media de 12 al mes) publicadas de marzo a julio de 2017 en B de Bulo, se observó el recurso de dos tipos de fuentes: mensajes procedentes de espacios de comunicación interactiva (WhatsApp, Telegram, Facebook o correo electrónico) y la búsqueda de contenidos que realizan los propios redactores, principalmente Jon Sedano como observador participante del estudio. Por la primera vía durante los cuatro meses de análisis se recibieron avisos de 18 personas diferentes en WhatsApp, una desde Telegram y cuatro vía Facebook. Algunos de esos usuarios remitieron varios mensajes con diferentes bulos, para conocer 
casi en tiempo real la veracidad del contenido o solicitando que se investigara y se desmintiera en la web en caso de ser falso.

\subsection{Rutinas productivas}

La bidireccionalidad de la comunicación con el usuario es clave para el éxito de la sección. Por ello cuando los lectores desean saber si es real el contenido que han enviado al medio, si el bulo ya está publicado en la web se les envía el enlace para que lean la noticia e incrementar el tráfico de la pieza con la seguridad de que el contenido resulta interesante para el usuario; si no se ha publicado pero se conoce que es un bulo debido a comunicados oficiales, se les hace saber, y por último, si no se tiene constancia del interés que pueda tener para la audiencia pero se considera un tema relevante, se verifica en la página. Para valorar si es relevante o no, se estudia si el tema ha tenido repercusión (buscando noticias al respecto en Internet o a través de redes sociales) o si es un caso aislado. Si la noticia puede llegar a interesar a una audiencia amplia, se comienza a trabajar en ella.

El 75\% del contenido publicado durante los cuatro meses de análisis fue propuesto directamente por periodistas, y un 25\% fue iniciativa de la audiencia: el 20\% llegó a través de WhatsApp mediante 18 personas diferentes, el $4 \%$ a través de Facebook, y solo una sugerencia llegó a través de Telegram.

Las 18 personas que se pusieron en contacto por WhatsApp enviaron un total de 34 avisos diferentes, de los que uno era publicidad de un grupo pornográfico y otro era el aviso de una próxima manifestación. De los 32 restantes, se publicaron siete noticias en $B$ de Bulo, divididas de la siguiente forma:

\begin{tabular}{|l|c|}
\hline Noticias publicadas en B de Bulo procedentes de WhatsApp & $\mathbf{N}^{\mathbf{o}}$ de avisos \\
\hline Furgoneta blanca que secuestraba niños en Málaga & 4 \\
\hline Página web que ofrecía un año de suscripción gratuita al servicio Netflix & 2 \\
\hline Mujer que secuestraba niños en las inmediaciones de Rincón de la Victoria & 1 \\
\hline Campaña solidaria de MRW para recopilar ropa con destino Portugal & 1 \\
\hline Actualización de Windows que escondía un virus & 1 \\
\hline Grupo de WhatsApp del DAESH del que es imposible salir & 1 \\
Aguja infectada con VIH en manguera de gasolinera & 1 \\
\hline
\end{tabular}

Tabla 1. Noticias publicadas procedentes de usuarios vía WhatsApp. Fuente: Elaboración propia

Desde Telegram, la persona que se puso en contacto envió dos mensajes: uno de ellos sobre una noticia ya publicada (fábrica de cerveza de Heineken en Sevilla con palomas succionadas) y otra de una niña desaparecida. En el caso de la primera se le envió el enlace a la noticia original, mientras que con la segunda se le agradeció el ponerse en contacto, pero no se publicó por afectar a una menor.

En cuanto a Facebook, las personas mensajeras avisaron de sucesos diferentes, de los que se publicaron dos en $B$ de Bulo: la banda que ataca a quienes les dan las luces en los vehículos y el nuevo control de alcoholemia a los copilotos. 
La ética profesional es uno de los parámetros que influye en el criterio de publicación de bulos. En este sentido, de las noticias recibidas por los usuarios, hubo dos que se descartaron publicar una vez investigadas. La primera era referente a una gasolinera de Málaga con agua en sus depósitos que había dañado a varios vehículos. En este caso se concluyó que era un bulo, pero debido a que el mensaje no había tenido gran repercusión, se estimó que su publicación haría más daño que beneficio a la empresa, por lo que solo se comunicó al lector que envió el mensaje que se trataba de un bulo. El segundo aviso era el caso de una niña que había sido secuestrada en México, y se descubrió que la niña ya estaba a salvo. A $B$ de Bulo llegaron cuatro mensajes desde WhatsApp sobre ella y uno desde Facebook, y se explicó a dichos usuarios que la niña ya estaba a salvo y que solo había desaparecido dos horas. De nuevo, no se consideró oportuno publicar la noticia en la web debido a que se trataba de una menor y ya estaba a salvo.

Finalmente, al teléfono de Diario Sur también han llegado por parte de la audiencia mensajes con bulos de forma puntual.

Gráfico 1. Procedencia de los bulos que aparecen en la sección. Fuente: Elaboración propia

— Método más utilizado para enviar información a B de Bulo

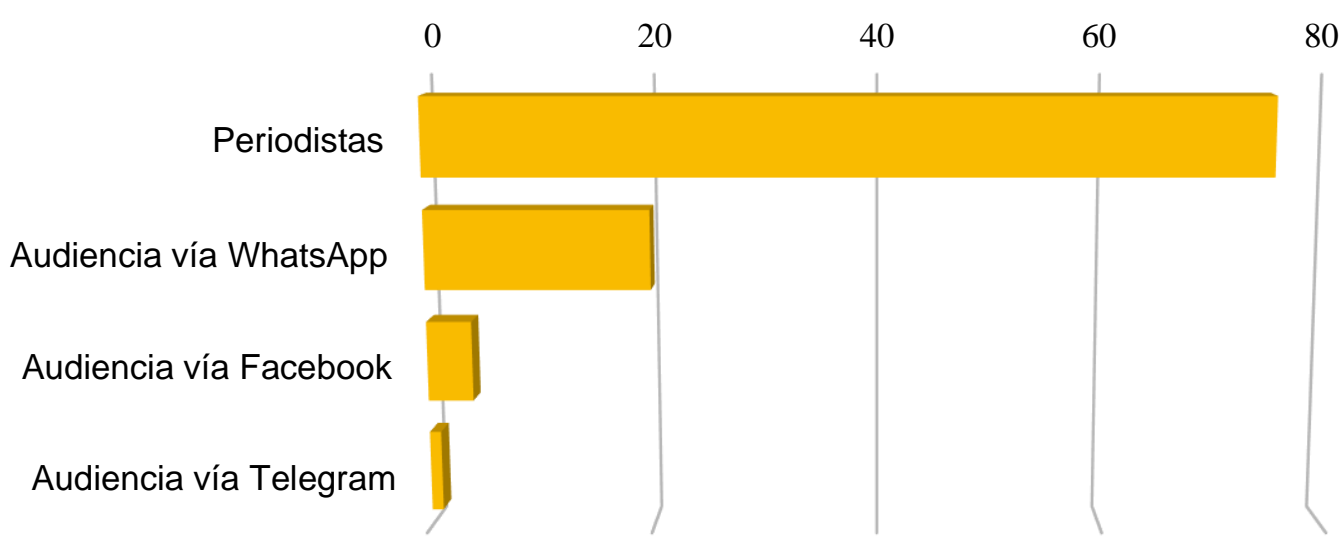

El resto de noticias que se han publicado en la sección fueron propuestas por el periodista. Para localizar dichos contenidos se articula la siguiente rutina: En primer lugar se realiza una búsqueda en Twitter con hashtags como \#bulo o \#stopbulos para conocer mensajes recientes donde los usuarios hablan de bulos. Simultáneamente, se sigue a varias cuentas oficiales que suelen hacerse eco de este tipo de publicaciones, como son los perfiles de la Policía Nacional, Guardia Civil, Tráfico, VOST (Equipo de voluntarios digitales en emergencias) o SOSdesaparecidos, entre otras.

Partiendo de este contenido, o el que se puede encontrar buscando noticias por la red que hagan uso del término 'bulo', así como analizando las principales noticias virales, se obtiene una base diaria para seleccionar potenciales contenidos de la sección. A pesar del volumen localizado en ocasiones, existe una limitación editorial en Diario Sur que consiste en que cada publicación debe espaciarse un mínimo de 48 horas de la siguiente, con el fin de no otorgar excesiva notoriedad a contenidos originalmente falsos en la portada del diario digital, lo que podría confundir al lector. Esta medida además favorece la correcta verificación de dichos contenidos. Otra restricción es que evitan contenidos vinculados con niños y con el mundo de la política. 


\subsection{Estrategias de verificación}

El periodista siempre ofrece argumentos, basados en hechos y datos, para justificar por qué un mensaje es falso. El proceso de verificación es diferente en cada noticia, aunque los pasos a seguir desde una perspectiva genérica son similares. Así, en primer lugar, se busca la fuente original para comprobar la veracidad de esta. En el caso de fotografías o vídeos, se extraen las imágenes y se utiliza la búsqueda nativa de Google para observar si aparecen noticias relacionadas.

En otras ocasiones se emplean palabras clave en varios idiomas para encontrar algún dato que pueda ser de ayuda. Por ejemplo, el vídeo sobre un aerogenerador que se rompía por la fuerza del viento en Cádiz, fue grabado en realidad en Italia, y posteriormente manipulado: se había cortado el inicio para no identificar el idioma y se había añadido ruido para ocultar la intervención oral original. Para localizar el origen de la pieza, se realizó una búsqueda de vídeos sobre aerogeneradores rompiéndose. $\mathrm{Al}$ no encontrar el vídeo, se extrajo un fotograma en la mejor calidad posible que al ser subido a Google devolvió una noticia similar. Siguiendo el rastro de esta noticia se dio con el vídeo en cuestión, que muchos medios situaban en Italia. Una vez obtenidas la localización y fechas exactas se hizo una búsqueda hasta dar con un medio relevante que publicó la noticia. Una vez verificado todo, se redactó el artículo en la sección aludiendo a la fuente original.

Prácticamente todos los contenidos son verificados acudiendo a la fuente original. Ésta permite comprobar la veracidad del hecho o la búsqueda de más datos que ayuden a determinar si es un bulo o no.

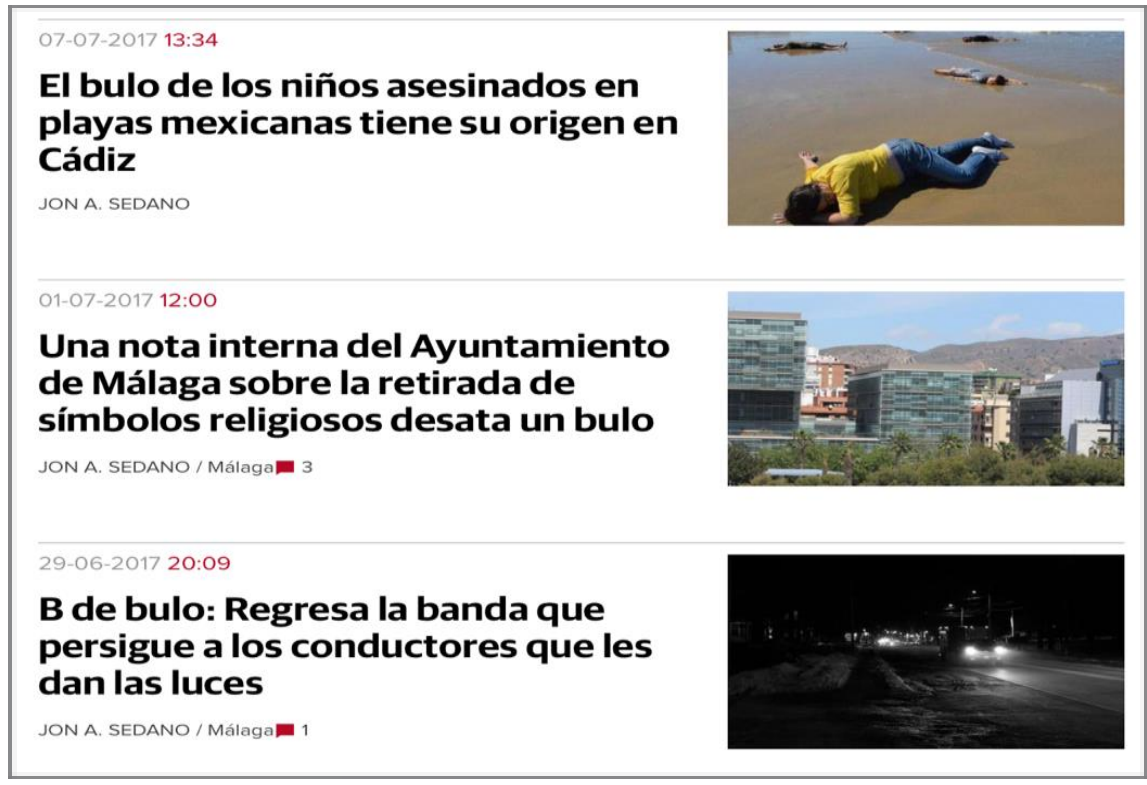

Otra forma de verificar una noticia falsa es utilizar el mensaje de una fuente oficial. En ese caso, se explica el bulo en sí y se contrasta con la información oficial que hay al respecto, añadiendo el enlace, tuit o la cita en el texto, para que los lectores puedan confirmar por sí mismos que se trata de una mentira.

Por último, en el caso de noticias locales, sobre todo sucesos, se contrastan con fuentes primarias que puedan tener acceso a dichos datos, como son la policía local o nacional, el servicio sanitario, etc. 
además de aquellas personas involucradas directamente en el tema, como ocurrió con la gasolinera. Para verificar aquella noticia, además de hablar con la propia estación y con otra persona de la empresa, se contactó con una gasolinera cercana de la competencia, con el sindicato de taxistas y con atención al consumidor de Málaga. Todos afirmaron que se trataba de un bulo. Por otra parte, los bulos que indican que han denunciado el secuestro de unos niños en una localidad y que la policía ya está trabajando en el tema, se resuelven contactando con la delegación de esa zona a fin de contrastar la veracidad del mensaje.

En cuanto al tiempo medio que exige la verificación, este varía en cada noticia. Las más rápidas de producir son aquellas en las que fuentes oficiales confirman o niegan un contenido; en esos casos se redacta la noticia explicando lo ocurrido y se acompaña del mensaje original de la fuente oficial. Si no hay datos oficiales al respecto, la verificación puede oscilar entre unas horas y un día.

\section{Tipología de las noticias publicadas en B de Bulo}

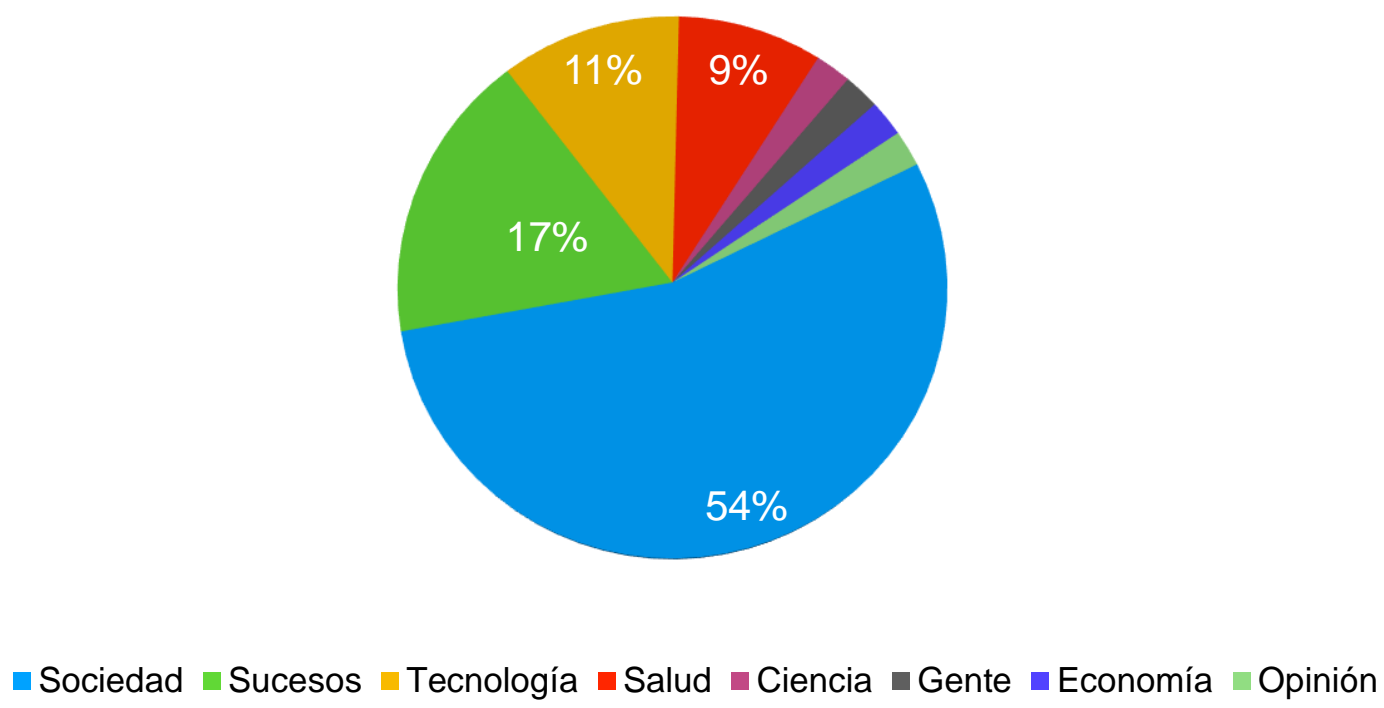

Portada de la sección B de Bulo. SUR

Excepcionalmente, en aquellos casos en los que se haya tenido que esperar la contestación de alguna parte implicada, se ha superado esa franja habitual.

\subsection{Las claves del éxito}

Ante la pregunta de por qué los usuarios comparten bulos, se han encontrado dos motivaciones: la primera es desacreditar a una empresa, persona o institución; y la segunda es crear una alarma social.

El miedo vende, y el análisis de las 50 noticias publicadas en B de Bulo durante sus primeros meses de existencia mostró que el 54 por ciento de ellas son contenidos sobre secuestros, muertes, fraudes, actividades terroristas, personas desaparecidas, accidentes, etc. Al mismo tiempo, se observó que solo el $14 \%$ de estos hechos estaban vinculados al entorno local, lo que permite al medio construir contenidos interesantes para otros entornos no necesariamente próximos (López, 1999). En este sentido, el periódico local genera contenido de interés global, conectado y abierto a las preferencias de los consumidores sin una limitación geográfica. 
A menudo los medios experimentan con la innovación sin obtener beneficio alguno. Sin embargo, la sección B de Bulo ha sido ampliamente aceptada por los lectores. El artículo más leído tuvo 214.192 visitas. De hecho, tres de estos artículos forman parte de una lista muy relevante: las cinco noticias más visitadas en la edición digital de Diario Sur durante 2017.

Todo el contenido se publica en cuentas de Facebook y Twitter, desarrolladas también por $B$ de Bulo. Después de monitorear las redes sociales, se observó que estas noticias han tenido más éxito en Facebook, obteniendo 1235 seguidores y 1200 "me gusta". En cuanto a las publicaciones más compartidas, con más clics y más reacciones, son las relacionadas con el secuestro.

\section{Conclusiones y discusión}

La tecnología no es neutral. Este rasgo obliga a que los periodistas deban desarrollar mecanismos para verificar todos los contenidos que reciben a través de los mensajes instantáneos de aplicaciones como WhatsApp o Telegram fundamentalmente por dos motivos: el primero, los medios tienen que crear puentes sólidos con el público para tratar de recuperar su confianza, colaborar eficazmente y crear compromiso y lealtad. Por otro lado, la mayoría de los medios de comunicación emplean correos electrónicos, mensajes de texto, mensajería instantánea, blogs y comentarios en sitios web, por lo que la posibilidad de una mala interpretación va en aumento. Los medios deben encontrar el equilibrio perfecto entre ambos escenarios.

En los últimos años, los beneficios de la comunicación low cost o de bajo coste se han reducido a describir la actividad de las audiencias en Twitter, Facebook, Instagram o Snapchat. Sin embargo, el periodismo actual se ve afectado por una sociedad sobreestimulada, abierta constantemente a nuevas experiencias. Los ciudadanos están interesados en la búsqueda de la novedad, que produce un golpe de dopamina. Paralelamente, cada alerta de WhatsApp representa un nuevo estímulo para los periodistas. Las aplicaciones de mensajería se han transformado en una fuente de información nueva y más rápida, y la actividad de la audiencia confirma su preferencia por estas plataformas, por lo que son canales muy eficaces para conectarse con el público. Paralelamente, esta comunicación privada y directa puede ser una intensa experiencia porque representa el perro guardián del perro guardián, y la fórmula que los medios tienen para recuperar la confianza de la audiencia.

Los ciudadanos tienen nuevos roles activos para ayudar a los medios a mejorar, a reducir la desinformación, a avisar de la circulación de las noticias falsas o los rumores. En este contexto, los periodistas deben recordar que obtener la primicia es un desafío, pero ser riguroso o preciso es una necesidad para sobrevivir en la práctica del periodismo de calidad.

Otra conclusión evidente tras el análisis de B de Bulo en Diario Sur es que WhatsApp es una herramienta ideal para medios pequeños o locales ya que no suelen tener la necesidad de construir listas con más de 253 usuarios, que es la limitación de la aplicación, y al ser la aplicación con mayor audiencia, con ella tienen la oportunidad de poder incrementar su público.

La inexistencia de investigaciones similares previas abre múltiples opciones de desarrollar comparativas con otras secciones de verificación de noticias en medios, plataformas destinadas de forma íntegra a contrastar bulos o un análisis cualitativo del comportamiento de la población ante las fake news en momentos de crisis sociales. 
- Investigación financiada: Este artículo es producto del proyecto de investigación titulado "La influencia de la audiencia en la innovación periodística: riesgos y oportunidades”, referencia CSO2015-64955-C4-3-R MINECO/FEDER. Financiado por el Programa Estatal de Investigación, Desarrollo e Innovación Orientada a los Retos de la Sociedad.

\section{Referencias bibliográficas}

Asociación de Internautas (2009): "Al 70\% de los internautas le cuesta distinguir un bulo de una noticia fiable". 02.09.2009. http://www.internautas.org/html/5699.html

Batra, B. (2016). "News Comunication Throught WhatsApp”. International Journal of Informative \& Futuristic Research, 3(10), 3725-3733.

Cadena Ser (2016): "Caso Nadia: Así se destaparon las mentiras del padre de Nadia". Cadena SER. 05.12.2016. http://cadenaser.com/programa/2016/12/05/hoy_por_hoy/1480934630_638829.html

Casal, César (2016): "La guerra de las noticias falsas". La Voz de Asturias. 04.12.2016. http://www.lavozdeasturias.es/noticia/asturias24/2016/12/04/guerra-noticiasfalsas/0003_201612G4P23992.html

CdPeriodismo (2013): "El tiempo se convierte en un problema ético cuando se prefiere lo rápido a lo correcto". Clases de Periodismo. 08.06.2013. http://www.clasesdeperiodismo.com/2013/06/08/eltiempo-se-convierte-en-un-problema-etico-cuando-se-prefiere-lo-rapido-a-lo-correcto/

Delclós, Tomás (2013): “Un tremendo error”. El País. 26.01.2013. http://elpais.com/elpais/2013/01/26/opinion/1359234476_377464.html

Editorial (2016): “Un grave error periodístico que no se debería repetir". El Mundo. 07.12.2016. http://www.elmundo.es/opinion/2016/12/07/58470d4b22601d62138b45c8.html

Fernández-Barrero, M. Ángeles, y Guirado, Víctor (2010): "Redes sociales, nuevas fuentes de información e investigación periodística”. En Comunicación y desarrollo en la era digital. Congreso AE-IC 3, 4 y 5 de febrero de 2010, ISBN 978-84-614-2818-2, p. 106.

https://idus.us.es/xmlui/handle/11441/31144

FM, Yúbal (2016): “2016: el año en el que las noticias falsas pusieron en jaque la credibilidad de Facebook". GenBeta. 17.12.2016. https://www.genbeta.com/a-fondo/2016-el-ano-en-el-que-lasnoticias-falsas-pusieron-en-jaque-la-credibilidad-de-facebook

García Santamaría, José Vicente (2010): “Crisis del periodismo de fuentes. Las prácticas del periodismo en España en el accidente de Spanair". Revista Latina de Comunicación Social, 65. http://www.revistalatinacs.org/10/art3/916_UC3M/38_Santamaria.html

Gottfried, Jeffrey, y Shearer, Elisa (2016): "News Use Across Social Media Platforms 2016". Pew Research Center. 26.05.2016. http://www.journalism.org/2016/05/26/news-use-across-social-mediaplatforms-2016/ 
Irujo, José María, y Elola, Joseba (2013): “Relato de un error de El País”. El País. 26.01.2013. https://elpais.com/internacional/2013/01/26/actualidad/1359234203_875647.html

Itkowitz, Colby (2016): "Fake news on Facebook is a real problem. These college students came up with a fix in 36 hours". The Washington Post. 18.11.2016.

https://www.washingtonpost.com/news/inspired-life/wp/2016/11/18/fake-news-on-facebook-is-areal-problem-these-college-students-came-up-with-a-fix/?noredirect=on\&utm term $=.79 \mathrm{~cd} 06582 \mathrm{c} 89$

Kawulich, Barbara B. (2006): "Participant Observation as a Data Collection Method". Forum Qualitative Sozialforschung / Forum: Qualitative Social Research, 6(2). http://nbnresolving.de/urn:nbn:de:0114-fqs0502430.

Jara, Juliana (2016): “El problema de las noticias falsas en Facebook". Digital Trends. 12.02. 2016. http://es.digitaltrends.com/sociales/problema-noticias-falsas-facebook/

Levenberg, Rubén (2011): "Reflexiones sobre la noticia en las redes sociales”. F@ ro: revista teórica del Departamento de Ciencias de la Comunicación, 13. http://web.upla.cl/revistafaro/n13/art23.htm

López García, Xosé (1999): "Estrategias locales en tiempos de globalización". Revista Latina de Comunicación Social, 21.

http://www.ull.es/publicaciones/latina/a1999dse/40xose.htm

López, Xosé; Rodríguez, Ana Isabel; y Álvarez, Lucía (2016): "El fact checking como reclamo y como servicio en los cibermedios. Análisis de las experiencias de 'The Washington Post' y 'The Guardian"'. Telos: Cuadernos de comunicación e innovación, 103. 74-83.

Mèdia.cat (2016): "Denzel Washington sobre les notícies falses i la responsabilitat dels mitjans". Mèdia.cat. 15.09.2016. https://www.media.cat/2016/12/15/denzel-washington-sobre-noticies-falsesresponsabilitat-dels-mitjans/

Miller, Jody y Barry, Glassner (2016): “The 'Inside' and the 'Outside': Finding Realities in Interviews“. En Silverman, David (ed.), Qualitative Research. Londres: Sage. p.51-66.

Negreira, María Cruz; López García, Xosé, y Lozano, Lara. (2017). Instant Messaging Networks as a New Channel to Spread the News: Use of WhatsApp and Telegram in the Spanish Online Media of Proximity. Recent Advances in Information Systems and Technologies. WorldCIST 2017. Advances in Intelligent Systems and Computing, 571, 64-72.

Newman, Nic; Levy, David; y Nielsen, Rasmus Kleis (2015): “Reuters Institute Digital News Report 2015". Reuters Institute for the Study of Journalism. Universidad de Oxford. Acceso el 13 de diciembre de 2016.

http://reutersinstitute.politics.ox.ac.uk/sites/default/files/research/files/Reuters\%2520Institute\%2520 Digital\%2520News\%2520Report\%25202015_Full\%2520Report.pdf

Paniagua, Francisco Javier (2017): "Recuperar la credibilidad". BEZ. Acceso 5 de enero de 2017. http://www.bez.es/721729736/recuperar-credibilidad.html

Projeto Credibilidade. https://www.credibilidade.org/trust-project 
Ramírez, Pedro J. (2013): “Ah, por cierto, ayer nos quisieron vender una foto de Chavez entubado. Dijimos no. Cuando la veáis en otro medio ya diréis si acertamos". [Actualización de estado de Twitter]. Acceso el 15 de diciembre de 2016.

https://twitter.com/pedroj_ramirez/status/294222368950329346?ref_src=twsrc\%5Etfw

Restrepo, Javier Darío (2016): "El papel de los gobiernos en el control de noticias falsas". Acceso 4 de enero de 2017. http://www.fnpi.org/consultorio-etico/consulta/1788

Rodríguez, Delia (2016): "Un problema del periodismo, siete de los periodistas y dos de los lectores". ElDiario.es. Acceso el 15 de diciembre de 2016. http://

www.eldiario.es/sociedad/problema-periodismo-periodistas-lectores_0_590741823.html

Sedano, Jon, y Palomo, Bella (2018). “Aproximación metodológica al impacto de WhatsApp y Telegram en las redacciones”. Hipertext.net, 16, 61-67. DOI: 10.31009/hipertext.net.2018.i16.10

Silverman, Craig. (2014): Verification Handbook. Reino Unido: European Journalism Centre. Acceso 14 de diciembre de 2016. http://verificationhandbook.com

Simón, Pedro. (2016): “Cuando no hay nada para Nadia". El Mundo. Acceso 18 de diciembre de 2016. http://www.elmundo.es/sociedad/2016/11/26/5838793bca47418e748b45e2.html

Torres, Jesús Erney (2004): “El rigor: garantía para los periodistas, credibilidad para los medios y esperanza para los ciudadanos". Palabra Clave, 11.

http://palabraclave.unisabana.edu.co/index.php/palabraclave/article/view/1301

Torres, Rafa (2007): "La verificación brilla por su ausencia en la red". Periodistas: revista de la Federación de Asociaciones de Periodistas de España, 11, pp. 35-37.

http://fape.es/file/1_periodistas11.pdf

\section{Cómo citar este artículo / Referencia normalizada}

B Palomo, J Sedano (2018): "WhatsApp como herramienta de verificación de fake news. El caso de B de Bulo". Revista Latina de Comunicación Social, 73, pp. 1384 a 1397.

http://www.revistalatinacs.org/073paper/1312/71 es.html

DOI: $10.4185 /$ RLCS-2018-1312

- En el interior de un texto:

...B Palomo, J Sedano (2018: 1384 a 1397) ...

o

...B Palomo et al, 2018 (1384 a 1397) ...

Artículo recibido el 14 enero de 2018. Aceptado el 16 de abril. Publicado el 25 de octubre de 2018 